\title{
Stimuli-Responsive Core-Shell Microcapsules with Tunable Rates of Release by Using a Depolymerizable Poly(phthalaldehyde) Membrane
}

\author{
Anthony M. DiLauro, ${ }^{\dagger, \S}$ Alireza Abbaspourrad, ${ }^{\ddagger}, \S$ David A. Weitz, ${ }^{\ddagger}$ and Scott T. Phillips ${ }^{* \dagger}$ \\ ${ }^{\dagger}$ Department of Chemistry, The Pennsylvania State University, University Park, Pennsylvania 16802, United States \\ ${ }^{\ddagger}$ School of Engineering and Applied Sciences, Department of Physics, Harvard University, Cambridge, Massachusetts 02138, United \\ States
}

Supporting Information

ABSTRACT: Flow-focusing microfluidic techniques were used to provide access to core-shell microcapsules in which the shell is composed of end-capped poly(phthalaldehydes) that depolymerize completely from head-to-tail in response to fluoride. Microcapsules made from these depolymerizable polymers provide an amplified response to the applied chemical signal, where the rate of the response can be tuned both by varying the length of the polymer and the thickness of the shell wall.

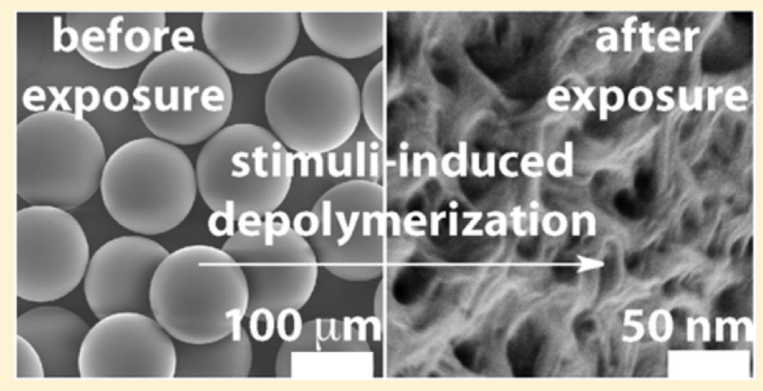

\section{INTRODUCTION}

Stimuli-responsive polymeric core-shell microcapsules are promising materials for controlled release applications. ${ }^{1-8}$ Capsules of this type typically release their contents either through induced chemical changes in the polymers that compose the shell or via bulk structural changes to the shell wall. These traditional triggered-release strategies provide responses that are nonamplified: i.e., a single membrane-signal interaction produces one small structural change in the shell wall, whereas release occurs only after many signals have reacted with and altered the shell wall. In contrast, a new release strategy has emerged where shell walls are formed from polymers that depolymerize continuously and completely from head-to-tail when an appropriate signal is detected by the polymer. ${ }^{1,9}$ This depolymerization reaction provides an amplified response that, in theory, increases the sensitivity of the capsules as well as their rate of response once the appropriate signal is detected. Despite these favorable attributes, however, only one example of a stimuli-responsive core-shell polymeric microcapsule made from a head-to-tail depolymerizable polymer has been demonstrated to date, ${ }^{10}$ with additional related examples demonstrated in responsive nanocapsules and micelles. ${ }^{11,12}$ Further development of this depolymerizable shell wall concept has been hindered by the limited number of polymers that are capable of depolymerizing from head-to-tail in response to a specific signal, as well as by substantial challenges in fabricating core-shell microcapsules using polymers that are primed to depolymerize.

In this article, we describe the use of flow-focusing microfluidic strategies for fabricating these types of depolymerizable core-shell microcapsules. ${ }^{13-15}$ This technique is highly reproducible, readily forms capsules that contain aqueous interiors, is capable of generating capsules that can be suspended in an aqueous solution that differs from the solution within the capsule, and does not require synthetic manipulation of the polymer for incorporation into the shell wall. Moreover, the fabrication technique is exceptionally mild and thus enables the formation of core-shell microcapsules using sensitive depolymerizable polymers such as poly(phthalaldehyde) (PPHA) (Figure 1). ${ }^{16,17}$ Herein we demonstrate these concepts

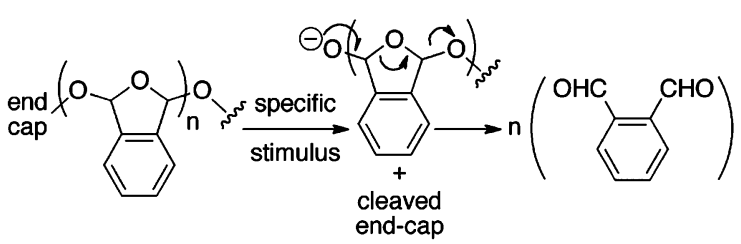

Figure 1. Mechanism of the head-to-tail depolymerization reaction when the end-cap on poly(phthalaldehyde) (PPHA) is cleaved via reaction with a specific stimulus. ${ }^{16}$

by fabricating model stimuli-responsive core-shell microcapsules using PPHA that contains a fluoride-responsive endcap. The end-cap controls the stability and reactivity of the PPHA polymer and thus enables release of the contents of the capsules selectively when exposed to fluoride (a model stimulus), even in aqueous media. We further demonstrate that the kinetics of cargo release depend both on the length of the polymer and the thickness of the shell wall. As a

Received: March 2, 2013

Revised: April 2, 2013

Published: April 16, 2013 
consequence, both the structural integrity (i.e., the thickness) and the rate of release can be tuned in a predictable way, such that capsules with desired structural and response properties can be fabricated easily.

Poly(phthalaldehyde) is a unique depolymerizable polymer and an interesting example for demonstrating the ability of flow-focusing to prepare core-shell microcapsules using sensitive depolymerizable polymers. The polymer is thermodynamically stable up to $\sim 150{ }^{\circ} \mathrm{C},{ }^{18}$ but it rapidly depolymerizes from head-to-tail when the bond between the polymer and the end-cap is broken (Figure 1). ${ }^{16}$ This chemical reaction forms a thermodynamically unstable hemiacetalterminated polymer (Figure 1), which has a ceiling temperature of $-40{ }^{\circ} \mathrm{C} .{ }^{16,18}$ Hence, once the end-cap is removed, the polymer depolymerizes quickly (seconds to minutes), selectively, and completely from head-to-tail, both in solution and in the solid state. ${ }^{16,17}$ The polymer also is sensitive to acid and base, which limits the number of techniques that can be used to fabricate microcapsules using PPHA. ${ }^{19}$

\section{RESULTS AND DISCUSSION}

Preparing End-Capped Poly(phthalaldehydes). To investigate the effect of membrane polymer molecular weight on the release kinetics, we prepared several lengths of the fluoride-responsive PPHA polymer using the anionic polymerization procedure shown in Figure 2. ${ }^{28,21}$ Each polymer was end-capped with 2-(methyoxypoly(ethylenoxy) ${ }_{6-9}$ propyl)dimethylchlorosilane, which reacts with fluoride.

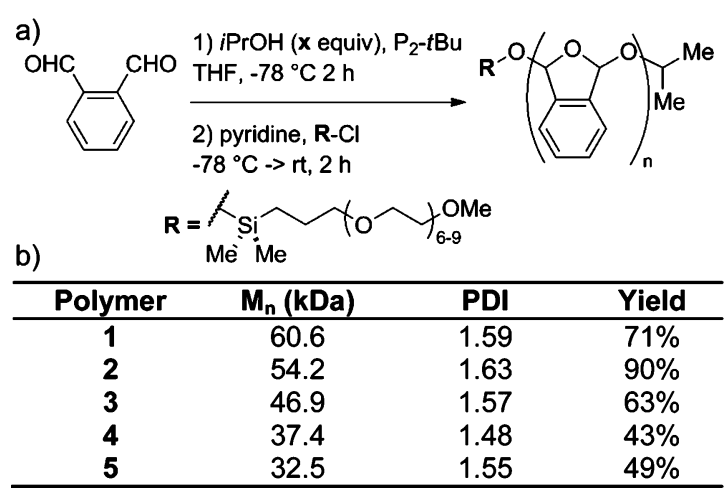

Figure 2. Synthesis of fluoride-responsive poly(phthalaldehydes). (a) The reaction conditions used to prepare the polymers. The equivalents of isopropanol used to initiate the polymerization reaction were varied to yield polymers with different number-average molecular weights $\left(M_{\mathrm{n}}\right) . \mathrm{R}-\mathrm{Cl}$ is the reagent used to incorporate the fluoride-responsive end-cap on the terminus of the polymer. (b) The polymers used in this study.

Characterizing the Depolymerization Response. Prior to generating fluoride-responsive core-shell microcapsules, we first determined the ability of the PPHA polymers in Figure 2 to depolymerize selectively in response to fluoride (Figure 3). Indeed, treatment of polymer $2\left(M_{\mathrm{n}}=54.2 \mathrm{kDa}\right)(1.6 \mathrm{mM})$ with $16 \mathrm{mM}$ tetrabutylammonium fluoride (TBAF) in 63:1 THF-aqueous buffer ( $\mathrm{pH} 7.1$ ) at $23{ }^{\circ} \mathrm{C}$ provided nearly complete depolymerization of 2 to monomer within $5 \mathrm{~h}$ (Figure 3a,b), whereas treatment with tetrabutylammonium chloride (TBAC) under identical conditions caused less than $4 \%$ depolymerization over $5 \mathrm{~h}^{22}$

Fabricating Core-Shell Microcapsules. We then fabricated core-shell microcapsules using the fluoride-responsive

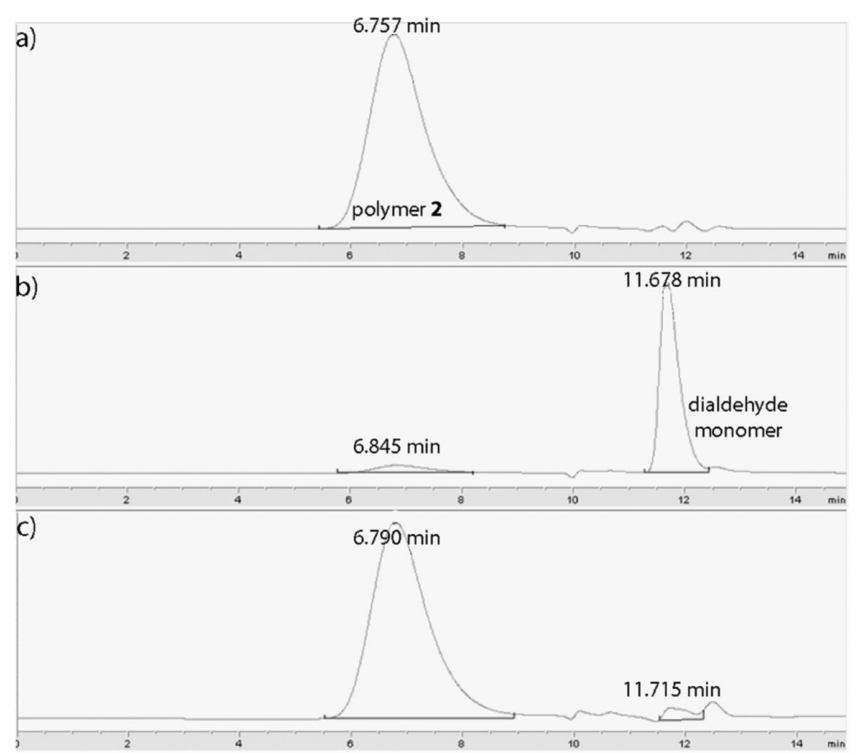

Figure 3. Demonstration that polymer 2 depolymerizes selectively when exposed to fluoride. Refractive index traces for 2 (a) before and (b) $5 \mathrm{~h}$ after exposure to $16 \mathrm{mM}$ TBAF in 63:1 THF-aqueous buffer $(\mathrm{pH} 7.1)$ at $23{ }^{\circ} \mathrm{C}$. (c) An experiment identical to part b, with the exception that 2 was exposed to TBAC instead of TBAF for $5 \mathrm{~h}$.

PPHA polymers by dissolving individual polymers in chloroform and then using microfluidic flow-focusing ${ }^{13-15,23}$ to encapsulate fluorescein isothiocyanate (FITC) labeled dextran (DEX) $(50 \mathrm{mg} / \mathrm{mL} ; 4 \mathrm{kDa} \mathrm{DEX})$ within the PPHA shell (Figure 4 and Movie S1, Supporting Information). Poly(vinyl
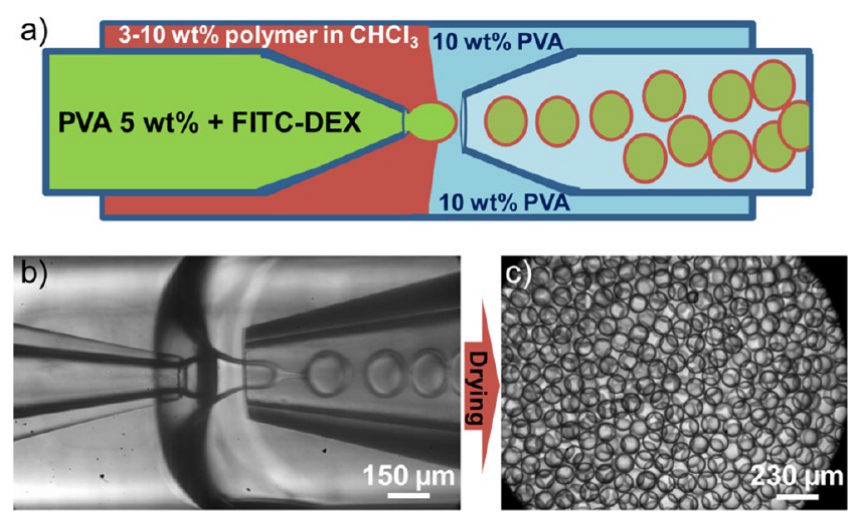

Figure 4. Fabrication of PPHA core-shell microcapsules using flowfocusing microfluidics. (a) Illustration of the double-emulsion technique used to fabricate the microcapsules. The color of the solution denotes the location of the solution in the microcapsules as follows: core solution (green), shell (red), and outer solvent (blue). (b and c) Optical microscope images showing (b) the generation of double-emulsion droplets of PPHA within the device, and (c) the capsules with consolidated membranes after evaporation of the $\mathrm{CHCl}_{3}$.

alcohol) (PVA) (5 wt \%) was included as well in the aqueous solution in the core of the capsule to balance the osmotic pressure between the interior and exterior of the capsule (the exterior solution was $10 \mathrm{wt} \%$ PVA in water). Control over the flow rate between the inner (core), middle (shell), and outer solutions and over the weight percent of polymer in chloroform allowed us to control the thickness of the shell walls of the capsules (see the Supporting Information for details) (Figure 
4b). Evaporation of the chloroform provided monodisperse capsules, as depicted in Figure 4c.

Characterizing the Microcapsules. Scanning electron microscope (SEM) images of capsules that had been freezedried reveal a homogeneous and smooth shell wall (Figure S1, Supporting Information). Manually crushing the capsules using a razor blade provided cross sections of the shell walls that enabled measurement of their thickness from the SEM images (Figure S2, Supporting Information). Microcapsules that were prepared using core-shell-outer solution flow rates of 600:700:8000 $\mu \mathrm{L} / \mathrm{h}$ and from a 10 wt \% solution of polymer $3\left(M_{\mathrm{n}}=46.8 \mathrm{kDa}\right)$ in chloroform, for example, had an average shell wall thickness of $1805 \mathrm{~nm} \pm 79 \mathrm{~nm}$ (average of 14 measurements).

Measuring the Responses of the Microcapsules. Capsules made using polymer $3\left(M_{\mathrm{n}}=46.8 \mathrm{kDa}\right)$ were tested for their ability to release FITC-DEX when exposed to aqueous fluoride. The capsules were dispersed in a solution containing $5 \%$ ethyl acetate in water and were further diluted with an equal volume of tetrabutylammonium fluoride (TBAF, $0.1 \mathrm{M}$ ) dissolved in 2:1 aqueous phosphate buffer (0.1 M, pH 7.1)THF (the final [TBAF] was $50 \mathrm{mM}$ ). We calculated the percentage of FITC-DEX that was released over time upon exposure of the capsules to aqueous fluoride (Figure 5k) by

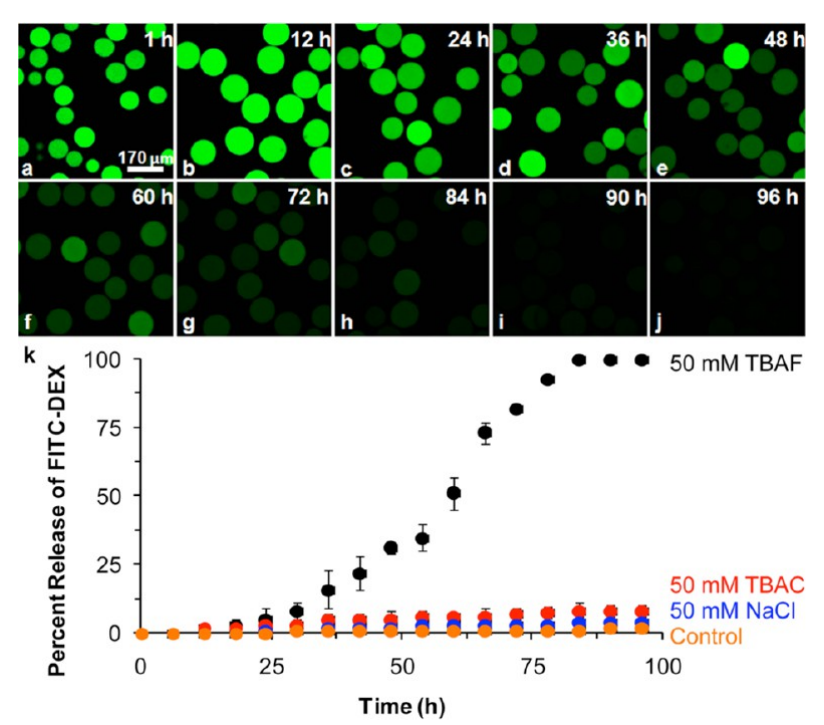

Figure 5. Controlled release of FITC-DEX from fluoride-responsive PPHA core-shell microcapsules (made from polymer 3) when exposed to $50 \mathrm{mM}$ aqueous fluoride. $(\mathrm{a}-\mathrm{j})$ Optical images and fluorescence measurements were acquired over $96 \mathrm{~h}$ for 100 capsules. The images show a subset of the capsules. (k) The percentage of FITC-DEX released from the capsules over time was calculated by comparison of the average fluorescent intensity of 100 capsules with a calibration curve generated using premade solutions of FITC-DEX. The data points represent the average of 100 measurements and the error bars reflect the standard deviations from these averages. The initial concentration of dye in each capsule was $50 \mathrm{mg} / \mathrm{mL}$. The microcapsules had an average shell wall thickness of $1805 \pm 79 \mathrm{~nm}$.

measuring the fluorescence intensity of the contents of 100 microcapsules (Figures $5 \mathrm{a}-\mathrm{j}$ ) and by comparing the average fluorescence values from these measurements with a calibration curve generated using solutions of FITC-DEX (Figure S3, Supporting Information). Figure $5 \mathrm{k}$ reveals that complete release of FITC-DEX occurred within $84 \mathrm{~h}$ of exposure to 50 $\mathrm{mM}$ aqueous fluoride; similar experiments using higher concentrations of fluoride show faster responses (Movie S2, Supporting Information).

Characterizing the Selectivity of the Capsules for Fluoride. The selectivity of the release reaction was verified using three control experiments that involved exposing the PPHA shell-wall microcapsules to (i) $50 \mathrm{mM}$ tetrabutylammonium chloride (TBAC) or (ii) $50 \mathrm{mM} \mathrm{NaCl}$ or (iii) simply immersing the microcapsules in the aqueous solution without adding a salt (this latter test was designed to examine whether differences in osmotic pressure inside versus outside the capsules caused release over the duration of the experiment). As shown in Figure 5k, negligible quantities of FITC-DEX were released over $96 \mathrm{~h}$ in all control experiments (i.e., TBAC $=8 \pm$ $2 \%$ release, $\mathrm{NaCl}=4 \pm 2 \%$, and solvent $=2 \pm 1 \%$ ), thus confirming the selective release of FITC-DEX from the model capsules when exposed to fluoride.

This selective response also is apparent when comparing SEM images of capsules that were exposed to TBAF or TBAC (Figure 6). Images $\mathrm{b}, \mathrm{d}$, and $\mathrm{f}$ in Figure 6, for example, reveal pinholes that developed over time in the shell wall of the capsule after exposure to fluoride for $48 \mathrm{~h}$ (Figure 6d) and $96 \mathrm{~h}$ (Figure 6f). In contrast, Figure $6 \mathrm{~h}$ shows that the surface of the PPHA microcapsule is essentially unaffected after being exposed to $50 \mathrm{mM}$ TBAC instead of TBAF for $96 \mathrm{~h}$.

This change in morphology for the TBAF example also provides a plausible explanation for the sigmoidal release curve observed in Figure 5k. The initial induction period likely occurs when small pinholes begin to develop in the shell wall that are too small for substantial release of the encapsulated FITC-DEX. Once the holes in the capsules grow, more end-caps on the polymers become accessible as the hole develops, and the dyelabeled polymer begins to escape rapidly, thus leading to a sharp increase in the rate of release observed in Figure 5k. The rate slows again after most of the dye has been released from the capsules.

Modulating the Rate of Release by Altering the Thickness of the Shell Wall. The rate of release of FITCDEX from the PPHA capsules can be tuned and controlled both by adjusting the thickness of the shell walls and by using polymers of different lengths to create the shell walls. To demonstrate the former capability, we formulated microcapsules with $100 \pm 19,650 \pm 44,1000 \pm 105$, and $1800 \pm$ $165 \mathrm{~nm}$ thick shell walls by changing the flow rates in the flowfocusing device during the fabrication process and by changing the weight percentage of polymer in the shell solution. When exposed to $50 \mathrm{mM}$ fluoride, the capsules with thinner shell walls released FITC-DEX faster than capsules with thicker shell walls (Figure 7): e.g., capsules with $100 \mathrm{~nm}$ thick shell walls released $90 \%$ of the encapsulated FITC-DEX $2.5 \times$ faster than capsules with $1800 \mathrm{~nm}$ thick shell walls. The caveat with this method of tuning the rate of release is that capsules with thinner shell walls are less robust and more prone to nonspecific release caused by mechanical forces than capsules with thicker shell walls.

Modulating the Rate of Release by Varying the Length of Poly(phthalaldehyde) Used in the Shell Wall. Microcapsules fabricated using different length PPHA polymers provide a second level of control over the rate of release of FITC-DEX (Figure 7). For example, capsules with $1800 \mathrm{~nm}$-thick shell walls made from polymer $5\left(M_{\mathrm{n}}=32.5\right.$ $\mathrm{kDa}$ ) released FITC-DEX $1.5 \times$ faster than capsules with 1800 $\mathrm{nm}$-thick shell walls made from polymer $1\left(M_{\mathrm{n}}=60.6 \mathrm{kDa}\right)$ (both capsules were exposed to $50 \mathrm{mM}$ TBAF in the standard 

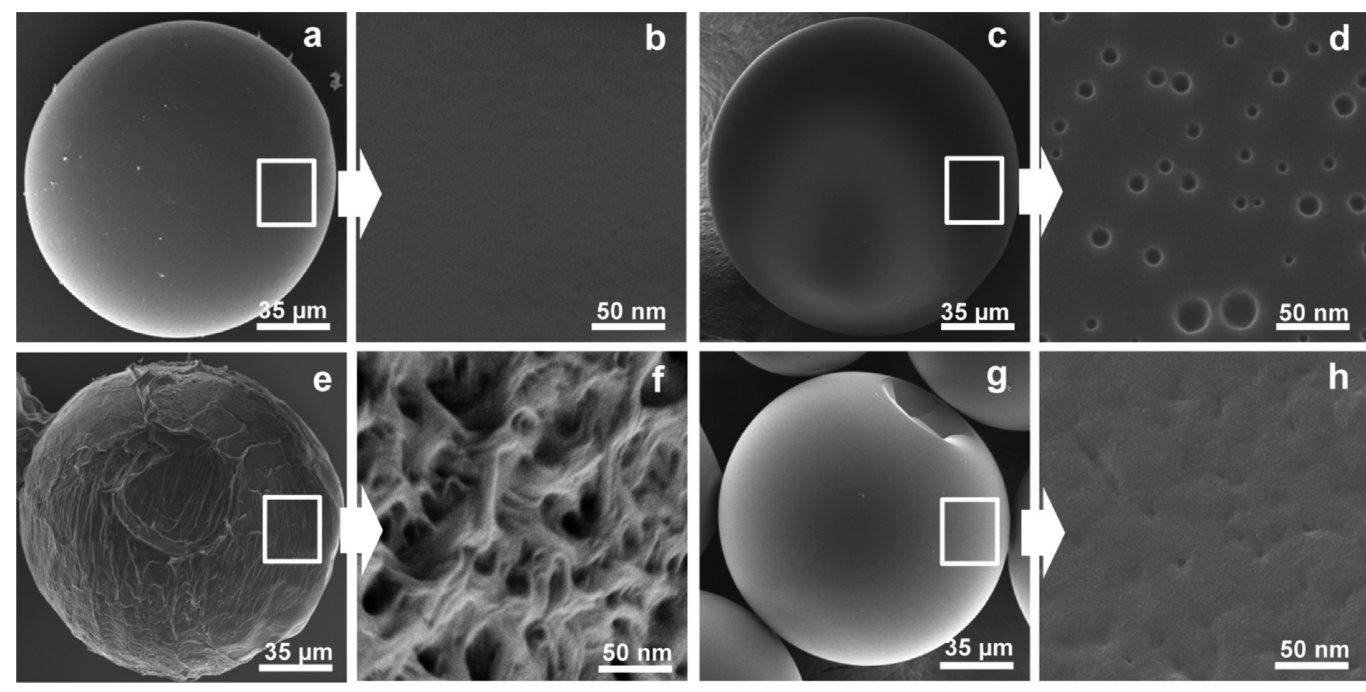

Figure 6. Changes in the morphology of the capsules over time when exposed to fluoride. The SEM images were acquired from freeze-dried samples at the following intervals after exposure to $50 \mathrm{mM}$ aqueous fluoride: ( $\mathrm{a}$ and $\mathrm{b})=0 \mathrm{~h}$; (c and d) $=48 \mathrm{~h}$; (e and f) $=96 \mathrm{~h}$. Images g and $\mathrm{h}$ were acquired $96 \mathrm{~h}$ after exposure of identical PPHA capsules to $50 \mathrm{mM}$ TBAC instead of TBAF. The capsules were made using polymer 3 and had an average shell wall thickness of $1805 \pm 79 \mathrm{~nm}$.

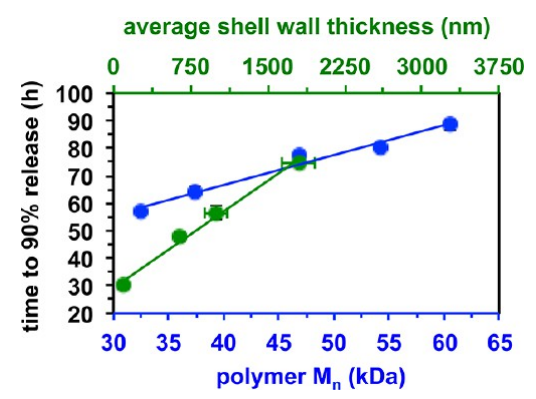

Figure 7. Tuning the rate of release from the microcapsule by varying the length of the polymer that is used to form the shell wall, and by varying the thickness of the shell wall. The values of Time to $90 \%$ Release were calculated from graphs of time vs percent release (Figures S4 and S5, Supporting Information). Polymer $3\left(M_{\mathrm{n}}=46.8\right.$ $\mathrm{kDa}$ ) was used to create the capsules for the shell wall thickness study, and polymers 1-5 were used to create $1800 \mathrm{~nm}$ thick shell walls for the polymer length study. The data points represent the average of measurements using 100 capsules. The $x$-axis error bars for average shell wall thickness represent the standard deviation in the shell wall thickness of six capsules. The $y$-axis error bars represent the standard deviation values determined from the line of best fit generated from sample sets (10 values) taken from each time point in Figures S4 and S5, Supporting Information, and are smaller than the data points.

aqueous solution). This linear correlation between polymer length and rate of response (Figure 7) suggests that more endcaps are available in the capsule shell wall to react with the stimulus (fluoride) when shorter polymers are used than longer polymers, thus enabling faster degradation of the shell wall and faster rate of release of FITC-DEX.

\section{CONCLUSION}

In conclusion, we have developed stimuli-responsive microcapsules that selectively release their contents through head-totail depolymerization of poly(phthalaldehyde) that forms the capsule membrane. The ability to predictably adjust both the thickness of the membrane and the length of the polymer within the membrane provides a useful level of control over the release event in the context of stimuli-responsive core-shell microcapsules composed of depolymerizable polymers. This level of control cannot be achieved easily using other fabrication techniques, and now makes possible the ability to rationally design depolymerizable core-shell microcapsules with a desired balance between rate of release and structural integrity (i.e., shell wall thickness).

Core-shell microcapsules made from poly(phthalaldehyde) (PPHA) may find uses in a variety of applications, but PPHA certainly is not the only depolymerizable polymer that should be compatible with the flow-focusing fabrication technique. Extension of this technique to other classes of depolymerizable polymers is currently underway and will be reported in due course. As more types of polymers become available, the value of head-to-tail depolymerizable core-shell microcapsules will become increasingly apparent, including their potential for rapid rates of release and their ability to respond to trace levels of an applied signal due to the amplification inherent in the depolymerization process. Moreover, capsules of this type should be capable of highly selective responses to a variety of external chemical and physical stimuli since only the composition of the end-cap on the polymer must be changed to create a capsule that responds to a new signal. Finally, the use of flow-focusing techniques enables rapid prototyping of microcapsules under mild conditions that enable chemically sensitive polymers (such as poly(phthalaldehydes)) to be used in the formation of responsive microcapsules.

\section{ASSOCIATED CONTENT}

\section{S Supporting Information}

Detailed synthetic procedures and methods for fabricating capsules, compound characterization data, tables of primary data, figures showing SEM images, FITC-DEX calibration curve, effect of the shell wall thickness and molecular weight on the rate of release, polymer GPC traces, and movies showing generation of droplets in the device and release of FITC-DEX. This material is available free of charge via the Internet at http://pubs.acs.org. 


\section{AUTHOR INFORMATION}

\section{Corresponding Author}

*(S.T.P.) E-mail: sphillips@psu.edu.

\section{Author Contributions}

${ }^{\S}$ A.M.D. and A.A. contributed equally to this work

\section{Notes}

The authors declare no competing financial interest.

\section{ACKNOWLEDGMENTS}

This work was supported in part by DARPA (HR0011-11-10007), NSF (CHE-1150969 and DMR-1006546), and the Harvard MRSEC (DMR 0820484), the Arnold and Mabel Beckman Foundation, the Camille and Henry Dreyfus Foundation, and Louis Martarano. S.T.P. acknowledges support from the Alfred P. Sloan Research Fellows Program.

\section{REFERENCES}

(1) Esser-Kahn, A. P.; Odom, S. A.; Sottos, N. R.; White, S. R.; Moore, J. S. Macromolecules 2011, 44, 5539-5553.

(2) Langer, R. Nature 1998, 392, 5-10.

(3) Meier, W. Chem. Soc. Rev. 2000, 29, 295-303.

(4) Matsusaki, M.; Akashi, M. Expert Opin. Drug Delivery 2009, 6, 1207-1217.

(5) Motornov, M.; Roiter, Y.; Tokarev, I.; Minko, S. Prog. Polym. Sci. 2010, 35, 174-211.

(6) Shchukin, D. G.; Grigoriev, D. O.; Möhwald, H. Soft Matter 2010, $6,720-725$.

(7) Johnston, A. P. R.; Such, G. K.; Caruso, F. Angew. Chem., Int. Ed. 2010, 49, 2664-2666.

(8) Brun-Graeppi, A. K. A. S.; Richard, C.; Bessodes, M.; Scherman, D.; Merten, O.-W. J. Controlled Release 2011, 149, 209-224.

(9) Peterson, G. I.; Larsen, M. B.; Boydston, A. J. Macromolecules 2012, 45, 7317-7328.

(10) Esser-Kahn, A. P.; Sottos, N. R.; White, S. R.; Moore, J. S. J. Am. Chem. Soc. 2010, 132, 10266-10268.

(11) DeWit, M. A.; Gillies, E. R. J. Am. Chem. Soc. 2009, 131, 1832718334.

(12) de Gracia Lux, C.; McFearin, C. L.; Joshi-Barr, S.; Sankaranarayanan, J.; Fomina, N.; Almutairi, A. ACS Macro Lett. 2012, 1, 922-926.

(13) Utada, A. S.; Lorenceau, E.; Link, D. R.; Kaplan, P. D.; Stone, H. A.; Weitz, D. A. Science 2005, 308, 537-541.

(14) Lorenceau, E.; Utada, A. S.; Link, D. R.; Cristobal, G.; Joanicot, M.; Weitz, D. A. Langmuir 2005, 21, 9183-9186.

(15) Shum, H. C.; Kim, J.-W.; Weitz, D. A. J. Am. Chem. Soc. 2008, 130, 9543-9549.

(16) Seo, W.; Phillips, S. T. J. Am. Chem. Soc. 2010, 132, 9234-9235.

(17) Zhang, H.; Yeung, K.; Robbins, J. S.; Pavlick, R. A.; Wu, M.; Sen, A.; Phillips, S. T. Angew. Chem., Int. Ed. 2012, 51, 2400-2404.

(18) Ito, H.; Willson, C. G. Polym. Eng. Sci. 1983, 23, 1012-1018.

(19) Kaitz, J. A.; Moore, J. S. Macromolecules 2013, 46, 608-612.

(20) DiLauro, A. D.; Robbins, J. S.; Phillips, S. T. Macromolecules 2013, DOI: $10.1021 / \mathrm{ma} 4001594$.

(21) Columbier, O.; Knoll, A.; Pires, D.; Gotsmann, B.; Duerig, U.; Frommer, J.; Miller, R.; Dubois, P.; Hedrick, J. L. Macromolecules 2010, 43, 572-574.

(22) In a previous study (ref 16) using an analogous silyl ether endcap, we used additional control studies to demonstrate that the endcap was responsible for the selective depolymerization reaction.

(23) Yow, H. N.; Routh, A. F. Soft Matter 2006, 2, 940-949. 